\title{
Editorial
}

Cristina Vassalle

\section{New biomarkers and traditional cardiovascular risk scores: any crystal ball for current effective advice and future exact prediction?}

https://doi.org/10.1515/cclm-2018-0490

The term "biomarker", which means "biological marker", refers to "any substance, structure, or process that can be measured in the human body" and "influences or predicts the incidence of outcome or disease" according to the definition of the World Health Organization [1].

Osteocalcin (OC), produced by osteoblasts, is traditionally considered a marker of bone formation, involved in the process of mineralization [2]. However, OC has attracted attention for its relationship with glucose metabolism and fat mass and its association with vascular calcification and atherosclerosis $[2,3]$.

The manuscript of $\mathrm{Xu}$ et al. [4] focuses on the role of OC for the identification of carotid intima-media thickness (C-IMT) over the atherosclerotic cardiovascular disease (ASCVD) risk score in a Chinese population $(n=618)$ of middle-aged and elderly men. Body mass index and glycated hemoglobin were identified as independent predictors for reduced OC levels, which confirms the active role of OC in cardiometabolic risk [4]. There is a higher recognition rate of elevated C-IMT when both low OC and moderate-to-high ASCVD risk are considered in comparison to considering a single parameter alone [4]. In particular, OC is independently and inversely associated with elevated C-IMT after being adjusted for the 10-year ASCVD risk score, and this relationship is especially evident in subjects with a moderate-to-high ASCVD risk [4].

In this context, a recent review and meta-analysis including 46 studies points out 26 positive, 17 negative and 29 neutral associations between markers of calcification or atherosclerosis and OC in blood, presence of OCpositive cells or OC histological staining. Authors did not conclusively identify a definitive association, although a consistent positive correlation was evident between the presence of OC-positive cells and histological staining with calcification and atherosclerosis [3]. Interestingly, variations related to ethnic differences appear important, as $37 \%$ of Asian studies reported negative relationships between $\mathrm{OC}$ and calcification or atherosclerosis, compared to $6 \%$ of European studies [3]. Such ethnic heterogeneity may be explained by the variation in body composition and genetic-related differences, as well as diet and sociodemographic factors. More generally, difficulties in the interpretation of the OC and atherosclerosis relationship may also be due to the measurement of total OC rather than its undercarboxylated and carboxylated forms, to the variety of studied patient cohorts (including kidney disease, diabetes or glucose intolerance, postmenopausal women and hypertension) and to the lack of studies primarily aiming to assess OC significance in atherosclerosis.

In the cardiovascular (CV) field, there are a few multifactorial integrated prediction algorithms for the estimation of CV risk (e.g. the Framingham Heart Study Risk Score), which stratify patients into low-, intermediateand high-risk categories [5]. Nonetheless, although much of CV risk may be attributed to traditional risk factors (e.g. smoking habits, diet or blood pressure) included in classical risk scores, overall CV risk is not entirely explained by traditional risk determinants [5]. Specifically, some events may also occur in subjects defined at low or intermediate risk because they present none or only one of the $\mathrm{CV}$ risk factors included in the traditional scores. Conversely, many high-risk individuals will not experience a CV event, even in the long term. These groups may be better classified according to a more adequate risk threshold in order to identify subclinical atherosclerosis signs at the earliest possible stage and, therefore, prioritize those who would need more urgent preventive strategies. Thus, the results discussed in the manuscript of Xu et al. support the interest of combining newly proposed CV risk biomarkers with conventional algorithms. Recently, several different biomarkers have been proposed, such as genetic, circulating and imaging biomarkers [6-9]. Genetic biomarkers reflect the potential of heritable profiling for $\mathrm{CV}$ disease, providing a tool for early individual diagnosis and prediction [6]. Interestingly, although association between candidate genes and atherosclerosis rarely gave consistent results in the past, recent data coming from both genomewide association studies and whole-genome sequencing 
studies show critical molecular targets, or pathways likely to block or delay atherosclerosis even from early stages [6]. Different imaging techniques have improved early detection of CV diseases, allowing a direct assessment and quantification of plaques, as well as of myocardial perfusion and metabolism alterations, although generally restricted to symptomatic patients [7, 8]. Instead, biochemical markers are easily measurable in blood or urine and can be the most direct and reliable expression of different phases of the disease, from very early processes, such as endothelial activation, to later stages when the injury is clearly established [9]. However, the added contribution of a single biomarker over the contribution estimated with the traditional algorithm is generally found rather limited [9]. Because of the poor individual predictive performance of single biochemical criterion, it has been proposed that the assessment of global risk may require the evaluation of multiple biomarkers in a more comprehensive panel [8]. However, actually tested "multimarker approaches" only slightly improve risk prediction [8]. Generally, biomarkers involved in known pathogenic pathways, mostly including inflammation and thrombosis, are included in these panels. The discrimination potential of this approach can still be improved, including selecting biomarkers showing a low degree of correlation, and as such capturing different levels of information [8]. Specifically, the use of an "independent-pathway" approach including a relatively small number of weakly interrelated (or totally unrelated) biomarkers, thus reflecting different pathophysiological events, could guarantee substantial gain [8, 10-12]. As an example, the VIenna and Ludwigshafen CAD (VILCAD) risk score, specifically tailored for patients with stable CAD, was modified with the inclusion of male gender, age, heart rate, left ventricular ejection fraction, $\mathrm{N}$-terminal pro b-type natriuretic peptide, glycated hemoglobin, renin, vitamin D-25(OH)D and cystatin [10]. The results of this modification were effective, with a net reclassification index (NRI: the sum of the net percentage of cases which correctly move in the higher risk and the net percentage of non-cases correctly reclassified in the lower risk group) of $14.9 \%(\mathrm{p}<0.001)$, compared to the original VILCAD score [10]. In fact, almost half of patients classified as low-risk by the original VILCAD score were upgraded to moderate risk, and about one third of patients, originally classified at moderate risk, were upgraded to the high-risk group [10]. In another study, the optimum 5-year CHD Risk Assessment (CHDRA) algorithm, which included age, sex, diabetes, family history of myocardial infarction, together with seven biomarkers (CTACK, Eotaxin, Fas Ligand, HGF, IL-16, MCP-3 and sFas), significantly improved "intermediate risk" classification by the Framingham reference model [11]. Specifically, the CHDRA reclassified $25.7 \%$ subjects with events and $17 \%$ without events, all of whom were initially classified as intermediate risk by the Framingham risk model, with a NRI corresponding to $42.7 \%(p<0.001)$ [11]. Moreover, the addition of a multimarker score (including $\mathrm{N}$-terminal pro-brain natriuretic peptide, C-reactive protein and sensitive troponin I) to a conventional risk model improved the 10-year risk estimation for CV events in two middle-aged European population-based studies (FINRISK97 and Belfast PRIME; 10,466 subjects) with net $6 \%$ of cases classified "up" and net $5 \%$ of non-cases classified "down" (NRI was estimated at $0.11, \mathrm{p}<0.001)$ [12].

Currently, further studies evaluating whether the addition of these biomarkers can provide substantial change to the predicted accuracy of traditional models are of critical importance. A biomarker is to be considered valuable when it leads to reclassification of an individual's risk towards an ameliorative management. NRI for each biomarker must therefore be carefully evaluated, also in regard to specific populations for which traditional scores may not optimally work (e.g. women, elderly population) $[13,14]$. In this context, OC could represent a promising candidate to better assess cardiometabolic risk. The article by $\mathrm{Xu}$ et al. is a first clue, and it is especially interesting for the proposed pathophysiological linkage associating OC and atherosclerotic processes. However, the criteria that must be fulfilled by new risk markers in order to improve risk scores for CVD are not completely satisfied by this work. In particular, some critical aspects need to be more carefully evaluated (e.g. type and number of patients enrolled, reported ROC analysis, NRI given, validation cohort, etc.).

It is possible that other biomarkers, some even still undiscovered, might be more important. Thus, in the process aimed to identify new biomarkers and evaluate their real clinical relevance, technical advances, such as genomics, proteomics, metabolomics and lipidomics, can be considered because although still in their developmental phase, they retain exceptional potential for a relatively rapid diffusion into clinical practice. Computational statistic tools may also permit the processing of large amounts of data to provide new prognostic models. Exploitation of all available tools and use of targeted stand-alone biomarkers and/or multimarker risk models in combination with modified traditional scores may maximize prevention and interventional strategies, approaching the reality of a biomarker-guided prevention and stratification in a near future. 
Acknowledgments: The authors wish to thank Dr. Laura Sabatino for editorial assistance.

Author contributions: The author has accepted responsibility for the entire content of this submitted manuscript and approved submission.

Research funding: None declared.

Employment or leadership: None declared.

Honorarium: None declared.

\section{References}

1. WHO International Programme on Chemical Safety Biomarkers in Risk Assessment: Validity and Validation. 2001. Available at: http://www.inchem.org/documents/ehc/ehc/ehc222.htm

2. Vassalle C, Maffei S, lervasi G. Biomarker validation - technological, clinical and commercial aspects. Bone remodelling biomarkers: new actors on the old cardiovascular stage. Weinheim, Germany: Ed Wiley-VCH Verlag GmbH \& Co. KGaA, Published Online: 27 Feb 2015, Chapter 7, pp. 107-46. ISBN: 9783527337194 Online ISBN: 9783527680658 DOI:10.1002/9783527680658.ch7.

3. Millar SA, Patel H, Anderson SI, England TJ, O’Sullivan SE. Osteocalcin, vascular calcification, and atherosclerosis: a systematic review and meta-analysis. Front Endocrinol (Lausanne) 2017;8:183.

4. Xu Y, Ma X, Xiong Q, Zhang X, Shen Y, Bao Y. Osteocalcin value to identify subclinical atherosclerosis over atherosclerotic cardiovascular disease (ASCVD) risk score in middle-aged and elderly Chinese asymptomatic men. Clin Chem Lab Med 2018;56:1962-9.

5. Alagona P Jr, Ahmad TA. Cardiovascular disease risk assessment and prevention: current guidelines and limitations. Med Clin North Am 2015;99:711-31.
6. Auer PL, Stitziel NO. Genetic association studies in cardiovascular diseases: do we have enough power? Trends Cardiovasc Med 2017;27:397-404.

7. Upadhyay RK. Emerging risk biomarkers in cardiovascular diseases and disorders. J Lipids 2015;2015:971453.

8. Wang TJ. Assessing the role of circulating, genetic, and imaging biomarkers in cardiovascular risk prediction. Circulation 2011;123:551-65.

9. De Backer G, Graham I, Cooney MT. Do novel biomarkers add to existing scores of total cardiovascular risk? Eur J Prev Cardiol 2012;19:14-7.

10. Kleber ME, Goliasch G, Grammer TB, Pilz S, Tomaschitz A, Silbernagel G, et al. Evolving biomarkers improve prediction of long-term mortality in patients with stable coronary artery disease: the BIO-VILCAD score. J Intern Med 2014;276:184-94.

11. Cross DS, McCarty CA, Hytopoulos E, Beggs M, Nolan N, Harrington DS, et al. Coronary risk assessment among intermediate risk patients using a clinical and biomarker based algorithm developed and validated in two population cohorts. Curr Med Res Opin 2012;28:1819-30.

12. Blankenberg S, Zeller T, Saarela O, Havulinna AS, Kee F, TunstallPedoe H, et al. Contribution of 30 biomarkers to 10 -year cardiovascular risk estimation in 2 population cohorts: the MONICA, risk, genetics, archiving, and monograph (MORGAM) biomarker project. Circulation 2010;121:2388-97.

13. Bambrick P, Tan WS, Mulcahy R, Pope GA, Cooke J. Vascular risk assessment in older adults without a history of cardiovascular disease. Exp Gerontol 2016;79:37-45.

14. Pop D, Dădârlat A, Zdrenghea D. Novel cardiovascular risk markers in women with ischaemic heart disease. Cardiovasc J Afr 2014;25:137-41.

Cristina Vassalle: Fondazione G. Monasterio per la Ricerca Medica e di Sanità Pubblica CNR-Regione Toscana, via Moruzzi, 1, 56124 Pisa, Italy, E-mail: cristina.vassalle@ftgm.it 